\title{
The Influence of Receptor-Mediated Interactions on Reaction-Diffusion Mechanisms of Cellular Self-organisation
}

\author{
Václav Klika • Ruth E. Baker • Denis Headon • \\ Eamonn A. Gaffney
}

Received: 22 July 2011 / Accepted: 7 October 2011 / Published online: 10 November 2011

(C) Society for Mathematical Biology 2011

\begin{abstract}
Understanding the mechanisms governing and regulating self-organisation in the developing embryo is a key challenge that has puzzled and fascinated scientists for decades. Since its conception in 1952 the Turing model has been a paradigm for pattern formation, motivating numerous theoretical and experimental studies, though its verification at the molecular level in biological systems has remained elusive. In this work, we consider the influence of receptor-mediated dynamics within the framework of Turing models, showing how non-diffusing species impact the conditions for the emergence of self-organisation. We illustrate our results within the framework of hair follicle pre-patterning, showing how receptor interaction structures can be constrained by the requirement for patterning, without the need for detailed knowledge of the network dynamics. Finally, in the light of our results, we discuss the ability of such systems to pattern outside the classical limits of the Turing model, and the inherent dangers involved in model reduction.
\end{abstract}

Keywords Reaction-diffusion · Self-organisation · Receptor-mediated patterning · Turing models

\footnotetext{
V. Klika $(\bowtie)$

Department of Mathematics, FNSPE, Czech Technical University in Prague, Trojanova 13, Prague, 120 00, Czech Republic

e-mail: klika@it.cas.cz

V. Klika

Institute of Thermomechanics, Academy of Sciences of the Czech Republic, Dolejskova 8, Prague, 180 00, Czech Republic

R.E. Baker · E.A. Gaffney

Centre for Mathematical Biology, Mathematical Institute, University of Oxford, 24-29 St Giles', Oxford, OX1 3LB, UK

D. Headon

The Roslin Institute and Royal (Dick) School of Veterinary Studies, University of Edinburgh, Edinburgh, UK
} 


\section{Introduction}

Ever since the rejection of the preformationist idea that minute animacules lay essentially fully formed within germ cells, the self-organisation of biological structure during development has puzzled and fascinated scientists. For instance, recent high throughput sequencing studies have emphasised that phenotypic diversity emerges from relatively similar genomes and proteomes, while cross-species genetic rescue studies (Leuzinger et al. 1998) have highlighted a universality of developmental processes via homologous pathways, suggesting that diversity instead arises from differences in regulatory DNA rather than protein coding sequence evolution (Alberts et al. 2002; Carroll 2005). This biological economy ensures that many aspects of metazoan development can be analysed from studies of fruit flies and nematode worms and, at the same time, motivates theoretical modelling to test and explore the detailed consequences of hypothesised universal developmental mechanisms.

One such common theme in developmental processes is the crucial role of cell-cell communication, in particular long-range range signalling, for orchestrating differential gene expression and biological morphogenesis over scales which are much larger than that of a cell. Numerous diffusing biochemical signals, known as morphogens, have been demonstrated as instigating cell fates in a spatially heterogeneous manner via a differential cellular response to concentration levels, durations, and gradients (Wolpert 2002; Gilbert 2006). While the characteristic lengthscale of morphogen signalling typically ranges from tens of microns to millimetres (Alberts et al. 2002; Gregor et al. 2005; Kicheva et al. 2007), and thus is insufficient at organ scales, complex structures can form via sequential induction, with a cascade of repeated cell fate differentiation at the morphogen scale inducing ever finer detail (Alberts et al. 2002; Wolpert 1994).

One of the best examples of a developing organism in which the mechanisms of spatial organisation have been defined is the Drosophila embryo. In this system a morphogen called bicoid is produced at the future anterior pole of the embryo and diffuses from this source to form a concentration gradient across much of the embryo. Bicoid protein acts in a concentration-dependent manner to control expression of gap genes, which refine the bicoid morphogen gradient and regulate the expression of pair rule genes. In this way, a concentration gradient of bicoid ultimately serves as a positional guide, instructing nuclei of their position relative to the anterior pole and, via differential interpretation, ultimately defines the 14 parasegments which emerge in the initially unsegmented embryo. However, in the Drosophila embryo no true symmetry ever exists to be broken as bicoid localisation to the future anterior pole is achieved during the formation of the egg by maternal inputs (Wolpert 2002; Gilbert 2006). Thus, by this morphogen gradient system positional information is elaborated, but importantly in the context of this work, it is not generated de novo.

A fundamentally different mode of pattern formation, and one which is capable of breaking symmetry without pre-existing positional information, has been known to be theoretically possible since Turing's (1952) seminal study. In particular, periodic patterns with a defined size and spacing can spontaneously emerge by the amplification of random molecular noise via a crucial combination of diffusion and biochemical reactions, in distinct contrast to elaboration of existing het- 
erogeneity via classical morphogen gradients, as with the bicoid system. The basic, or vanilla, representation of Turing's concept requires two morphogens, one of which, the inhibitor, diffuses rapidly and exhibits self-antagonism while the other, the activator, diffuses slowly and is auto-catalytic (Gierer and Meinhardt 1972; Segel and Jackson 1972). A further requirement for self-organisation via the Turing mechanism is that the activator upregulates the inhibitor, which in turn down regulates the activator, or the converse cross-morphogen interactions. This elegant and economical mechanism has driven numerous experimental studies (e.g. Miura and Shiota 2000a, 2000b; Miura et al. 2006; Harris et al. 2005; Chen and Schier 2002; Sakuma et al. 2002; Solnica-Krezel 2003) which suggest that Turing-like morphogen interactions and patterns can occur in developmental systems, though the context often concerns refining, rather than initiating, spatial heterogeneity. As one example, hair follicle and feather patterning studies highlight numerous large scale features that are characteristic of Turing's mechanism, including the insertion of new pattern elements as the field is stretched (Davidson 1983), and transitions between spotted and striped patterns upon experimental manipulation (Mou et al. 2011; Lin et al. 2009). However, a direct verification of Turing's mechanism at the level of molecular details has remained elusive.

One biological feature lacking from Turing's model is the inclusion of cell-level detail. The compartmentalisation of tissues into discrete cells necessitates complex modes of intercellular communication and these must be addressed to bridge the gap between theoretical and experimental studies. Intercellular signalling, known to be the key driver of vertebrate development, as evidenced by innumerable transplantation and grafting experiments (Wolpert 2002; Gilbert 2006), typically requires a chain of signalling molecules acting in a pathway to receive and amplify signals present in the extracellular space (Lodish et al. 2007). Diffusion through a tissue is limited to extracellular molecules secreted from cells, or those passing though cellcell junctions, while the majority of proteins involved in intercellular signalling are restricted to the cell in which they are synthesised. Thus, translating Turing's two morphogen system to a biological context suggests that "activator" and "inhibitor" functions are each likely to involve a pathway composed of a chain of transiently interacting proteins, with the majority of these proteins not diffusing beyond the plasma membrane and thereby acting in a strictly cell autonomous manner. Even when regarded as pathways rather than individual chemical species, the regulatory interactions between activator and inhibitor described above should hold for the operation of a Turing system, though the precise points within a pathway at which the reciprocal activatory-inhibitory regulatory steps actually occur may be on either diffusing or non-diffusing species. The latter typically constitute the majority of components of intercellular signalling pathways, and the preponderance of genes encoding these non-diffusing entities suggests that much of the reciprocal activator-inhibitor regulatory interaction is likely to occur directly on non-diffusible species.

There are also theoretical difficulties, at least with the vanilla Turing mechanism. In particular, it is notoriously sensitive to changes in molecular parameter values and to variations in initial conditions. This is inappropriate given the constraints of development, as first noted in the context of initial conditions where small fluctuations could induce different outcomes (Bard and Lauder 1974). How- 
ever, domain growth has since been theoretically demonstrated as a potential mechanism to alleviate such sensitivity by conferring an evolving geometric constraint which induces a robust, sequential, instigation of self-organisation (Crampin et al. 1999). Such geometrical constraints may also ameliorate concerns about the sensitivity of Turing's mechanism to domain boundary perturbations (Bunow et al. 1980) though this is unexplored at present. However, the theoretical sensitivity of Turing's mechanism with respect to gene expression dynamics (Gaffney and Monk 2006; Seirin-Lee and Gaffney 2010; Seirin-Lee et al. 2010, 2011) defies the classical stabilising mechanism of growth though the prospect of amelioration via cellular, chemotactic, or feedback dynamics within networks of interacting of morphogens and signalling pathways remains to be considered.

More generally, this highlights that generalisations of Turing's mechanism, with the spontaneous emergence of self-organisation driven by fluctuations, is underexplored in biological networks as opposed to two-species systems. Mathematical studies have been limited to the derivation of conditions for self-organisation given three interacting morphogens or recursive conditions for networks or larger number of molecular components (White and Gilligan 1998; Satnoianu et al. 2000; Qian and Murray 2001), with relatively limited numbers of modelling studies that also include other mechanisms, such as haptotaxis and developmental waves (e.g. Lin et al. 2009; Hentschel et al. 2004; Alber et al. 2008).

However, advances within developmental molecular biology have explicitly highlighted in-vivo biological self-organisation morphogen networks in hair follicle and feather bud patterning with features of the long-range activation and short-range inhibition that characterise the vanilla Turing mechanism (Jung et al. 1998; Mou et al. 2006, 2011). Such features appear to be supplemented and regulated by morphogenreceptor interactions. However, the receptors are not subject to transport on the observed wavelengths of periodic patterning, which must be much larger than cellular scales since both feather and hair primordia contain many cells at their inception, with multiple cell diameters intervening between these pattern elements (see Sengel 1990 or Schmidt-Ullrich and Paus 2005 for reviews).

Nonetheless, a fundamental study of receptor dynamics within the context of Turing's mechanism is underexplored in that traditionally all species are assumed to be subject to long-range transport across the domain. In contrast receptors, being constrained to an individual cell, should be treated as non-diffusing species; such generalisations of the standard Turing model could also be considered in the context of intracellular proteins. Ermentrout and Lewis's (1997) study considered only two non-diffusing species together with one diffusing biochemical, in ecological and neurological contexts. This is too restrictive for the interactions of multiple diffusing activatory and inhibitory morphogens, such as those found in Mou et al.'s (2006) model, for example. A more recent investigation by Rauch and Millonas (2004) was considered in the context of receptor and protein dynamics, but introduced a small diffusion rate for intracellular proteins to avoid "mathematical difficulties" with a biological justification of cell membrane leakiness. However, transmembrane receptors and intracellular signal transducing proteins function only within specific subcellular compartments (e.g. the plasma membrane, the cytoplasm or the nucleus) and any leaked receptor protein would not have a signalling function in the extra- 
cellular space. Thus, below we instead utilise the complications associated with nondiffusing species within biological pattern formation models containing multiple diffusing morphogens, enabling a restriction of possible interaction networks.

Our first objective is therefore to explore how non-diffusing receptor dynamics within networks of receptors and diffusing morphogens impact the conditions for the emergence of self-organisation. Further, we will also specifically explore Mou et al.'s (2006) model for hair follicle patterning as this simple network offers a well motivated and tractable case study for assessing the extent to which features of the classical Turing model are retained by more complex interacting systems with receptors. In particular, our second objective will be to assess whether morphogen and receptor interaction networks can be constrained by the requirement of self-organisation in a biologically relevant setting. In turn, this will highlight the extent to which analytical methods can inform our understanding of more complex morphogen and receptor systems of patterning formation based upon Turing's concepts, including the extent to which they can be represented by the vanilla, two-species, Turing model.

We begin, in Sect. 2, by summarising the requirements for a Turing instability in networks of diffusible morphogens, before considering receptor dynamics in detail, whereby the diffusion coefficients of some network species are set to zero. In Sect. 3, we then explore conditions for self-organisation for Mou et al.'s model in detail, before discussing our findings and drawing conclusions in Sect. 4.

\section{A Linear Stability Analysis of Patterning in Multi-species Models}

Given a system of $n$ interacting biochemical species, we use $\mathbf{u}(\mathbf{x}, t)$ to denote the vector of chemical concentrations $\left(u_{1}(\mathbf{x}, t), u_{2}(\mathbf{x}, t), \ldots, u_{n}(\mathbf{x}, t)\right)$. In this case, the evolution of chemical concentration over time can be derived from conservation principles and shown to be given by the following system of reaction-diffusion equations:

$$
\frac{\partial}{\partial t} \mathbf{u}=\mathbf{D} \nabla^{2} \mathbf{u}+\mathbf{f}(\mathbf{u})
$$

where the vector $\mathbf{f}(\mathbf{u})$ describes the interactions taking place between biochemicals (including production and decay) and the diagonal matrix $\mathbf{D}$ consists of the constant diffusion coefficients $D_{j}, j \in 1, \ldots, n$ for the $n$ biochemicals. A homogeneous steady state, $\mathbf{u}^{*}$, is assumed to exist and is given by $\mathbf{f}\left(\mathbf{u}^{*}\right)=\mathbf{0}$. Boundary conditions and initial conditions are also required; we assume the former are zero flux and the latter are perturbations of the homogeneous steady state.

Standard linear analysis (Murray 2003) of the system about the homogeneous steady state can be used to test for linear instability. Perturbing about $\mathbf{u}^{*}$ by letting $\mathbf{u}=\mathbf{u}^{*}+\tilde{\mathbf{u}}$ where $|\tilde{\mathbf{u}}| \ll 1$ gives rise to the linearised system

$$
\frac{\partial}{\partial t} \tilde{\mathbf{u}}=\mathbf{D} \nabla^{2} \tilde{\mathbf{u}}+\mathbf{J} \tilde{\mathbf{u}}
$$


where the matrix $\mathbf{J}$ is the matrix of partial derivatives

$$
J_{i j}=\frac{\partial f_{i}}{\partial u_{j}},
$$

evaluated at the steady state $\mathbf{u}^{*}$.

Let $w_{k}(\mathbf{x})$ denote the eigenmodes of the Laplacian operator, $\nabla^{2}$, given zero flux boundary conditions. Thus, by definition,

$$
\nabla^{2} w_{k}=\eta_{k} w_{k}
$$

and, noting the boundary conditions, one can show that $\eta_{k} \leq 0$ is real (Baker et al. 2008); hence, we denote it by $-k^{2}$. Furthermore, only a countably infinite set of values of $k^{2}$ are permitted, as illustrated by the simple case of a one dimensional domain, $x \in[0, L]$, with zero flux boundary conditions where

$$
w_{k}(x)=\cos \left(\frac{k \pi x}{L}\right), \quad k \in\{0,1,2, \ldots\} .
$$

This form of $w_{k}$ also highlights that $k$ can be considered to correspond to the wavenumber of the eigenmode.

Seeking solutions in terms of series expansions of the eigenmodes, and invoking linearity to consider each eigenmode individually, leads one to consider the form

$$
\tilde{\mathbf{u}}=\mathbf{a} \exp (\lambda t) w_{k}(\mathbf{x})
$$

with $\mathbf{a} \neq \mathbf{0}$ constant and $\lambda$ in general complex. One can readily deduce that (2) subsequently demands

$$
\left(\lambda \mathbf{I}+k^{2} \mathbf{D}-\mathbf{J}\right) \mathbf{a}=0,
$$

which gives rise to the dispersion relation:

$$
p\left(\lambda, k^{2}\right)=\operatorname{det}\left(\lambda \mathbf{I}+k^{2} \mathbf{D}-\mathbf{J}\right)=0 .
$$

It is often convenient to interpret (8) as defining $\lambda_{*}\left(k^{2}\right)$, the roots of the dispersion relation for $\lambda$ as a function of $k^{2}$, via the implicit function theorem. Note that $\operatorname{Re}\left(\lambda_{*}\left(k^{2}\right)\right)<0$ corresponds to linear stability for wavenumber $k$ while $\operatorname{Re}\left(\lambda_{*}\left(k^{2}\right)\right)>0$ corresponds to linear instability.

A Turing instability requires stability in the spatially homogeneous system, $k^{2}=$ 0 , as only spatially constant perturbations are allowed in this case, and instability for some $k^{2}>0$, that is also minus an eigenvalue of the Laplacian. Numerical simulations consistently indicate that linear stability is sufficient to pinpoint when a Turing instability occurs, despite the fact dynamical systems can exhibit more complex nonlinear behaviours (Murray 2003).

However, note that caution is required in the case of Turing-Hopf instabilities, where

$$
\operatorname{Re}\left(\lambda_{*}\left(k^{2}\right)\right)>0, \quad \operatorname{Im}\left(\lambda_{*}\left(k^{2}\right)\right) \neq 0,
$$

as the concomitant oscillations necessitate a further fast-acting mechanism to arrest the temporal oscillations once the system is away from the homogeneous steady state. 


\subsection{Behaviour of the Dispersion Relation at Large Wavenumbers}

An important consistency constraint for Turing patterning is that the system is stable for sufficiently large wavenumbers: otherwise, arbitrarily small wavelengths can destabilise the system at lengthscales smaller than the cell, leading to a breakdown in the continuum approximation. This constraint is automatically satisfied for twospecies Turing models, as can be deduced from textbook analyses (Murray 2003). However, it has not been considered in general, as illustrated in the related context of mechanochemical models of vasculogenesis (Moreo et al. 2010). In particular, the loss of stability at large wavenumbers was observed to agree with the inexorable breakdown of continuum theory as eigenmodes of smaller and smaller wavelengths manifested within the solution. As the continuum approximation lost validity, heterogeneity on increasingly smaller lengthscales would lead to an emergence of salt and pepper patterning on the cellular scale, though whether such dynamics continues to emerge and stabilise as the continuum approximation becomes invalid requires a discrete framework and is unexplored.

For patterning on lengthscales much larger than the cell, this behaviour is not relevant and leads to the constraint of stability at large wavenumbers. Given this is a restrictive and informative constraint, we therefore firstly explore the potential for instabilities at arbitrarily high values of the wavenumber, or equivalently $k^{2}$, in Turing networks of diffusible morphogens.

\subsubsection{Large Wavenumber Behaviour of the Dispersion Relation for Networks of Diffusible Morphogens}

Given all species diffuse, we have that $\operatorname{det}\left(k^{2} \mathbf{D}\right) \neq 0$. In this case the dispersion relation, (8), gives

$$
\begin{gathered}
\operatorname{det}\left(k^{2} \mathbf{D}\right) \operatorname{det}\left[\left(\frac{\lambda}{k^{2}} \mathbf{D}^{-1}+I\right)-\frac{1}{k^{2}} \mathbf{D}^{-1} \mathbf{J}\right]=0 \\
\Longleftrightarrow \operatorname{det}\left[I+\frac{1}{k^{2}}\left(\lambda \mathbf{D}^{-1}-\mathbf{D}^{-1} \mathbf{J}\right)\right]=0,
\end{gathered}
$$

which can be rewritten as

$$
1+\frac{1}{k^{2}}\left[\lambda \sum_{j=1}^{n} \frac{1}{D_{j}}-\sum_{j=1}^{n} \frac{1}{D_{j}} J_{j j}\right]+\mathcal{O}\left(\frac{1}{k^{4}}\right)=0 .
$$

Firstly, we note that if $|\lambda| \sim \mathcal{O}(1)$ there is no solution for $k^{2} \gg 1$ in the (biologically relevant) case that $J_{i j}$ is bounded. Secondly, if $|\lambda| \gg \mathcal{O}\left(k^{2}\right)$ there is no solution for $k^{2} \gg 1$ as we have (denoting $\varepsilon=k^{2} / \lambda \ll 1$ )

$$
\begin{aligned}
0 & =\operatorname{det}\left(I+\frac{1}{\varepsilon} \mathbf{D}^{-1}-\frac{1}{k^{2}} \mathbf{D}^{-1} \mathbf{J}\right) \\
& =\frac{\operatorname{det} D^{-1}}{\varepsilon^{n}}\left[\operatorname{det}\left(I+\varepsilon \mathbf{D}-\frac{\varepsilon}{k^{2}} \mathbf{J}\right)\right]
\end{aligned}
$$




$$
\begin{aligned}
& \approx \frac{\operatorname{det} D^{-1}}{\varepsilon^{n}}\left(1+\varepsilon \operatorname{tr} \mathbf{D}-\frac{\varepsilon}{k^{2}} \operatorname{tr} \mathbf{J}\right) \\
& \approx \frac{\operatorname{det} D^{-1}}{\varepsilon^{n}} \neq 0 .
\end{aligned}
$$

Now consider the case where the roots of the dispersion relation are such that $\lambda_{*}\left(k^{2}\right)=\mu k^{2}$ with $\mu \sim \mathcal{O}(1)$. Then (10) gives

$$
\operatorname{det}\left(I+\mu \mathbf{D}^{-1}-\frac{1}{k^{2}} \mathbf{D}^{-1} \mathbf{J}\right)=\operatorname{det}\left(I+\mu \mathbf{D}^{-1}\right)+\mathcal{O}\left(\frac{1}{k^{2}}\right) .
$$

Recalling that $\mathbf{D}$, and hence its inverse, are diagonal we have that when $k^{2}$ is sufficiently large equation (13) has roots $\mu=-D_{i}, i \in 1, \ldots, n$, which gives the corresponding roots of the dispersion relation:

$$
\lambda_{*}\left(k^{2}\right) \approx-k^{2} D_{1},-k^{2} D_{2}, \ldots,-k^{2} D_{n},
$$

when $k$ is sufficiently large and $D_{i}>0, i \in 1, \ldots, n$.

In summary, the requirement of stability at sufficiently large wavenumbers is guaranteed in Turing models when all species diffuse.

\subsubsection{The Inclusion of Receptors and the Large Wavenumber Behaviour of the Dispersion Relation}

Suppose now that $m<n$ species within the model represent receptors, which therefore do not undergo transport on the scale of long-range cell signalling but instead are restricted to a cell. Thus, they have zero diffusion coefficients and, without loss of generality, we take $D_{1}, \ldots, D_{m}=0$.

First, we consider the dispersion relation, (8), which takes the form

$$
0=\operatorname{det}\left[\lambda \mathbf{I}+k^{2} \mathbf{D}-\mathbf{J}\right]=
$$$$
\operatorname{det}\left(\begin{array}{c|c|c||c|c|c|c}
\lambda-J_{11} & -J_{1, m} & -J_{1, m+1} & -J_{1, m+2} & -J_{1, n} \\
-J_{21} & & -J_{2, m} & -J_{2, m+1} & -J_{2, m+2} & -J_{2, n} \\
-J_{31} & & -J_{3, m} & -J_{3, m+1} & -J_{3, m+2} & & -J_{3, n} \\
\vdots & \ldots & \vdots & \vdots & \vdots & \cdots & \vdots \\
-J_{m, 1} & \lambda-J_{m, m} & -J_{m, m+1} & -J_{m, m+2} & -J_{m, n} \\
\hline \hline-J_{m+1,1} & & -J_{m+1, m} & \lambda+k^{2} D_{m+1}-J_{m+1, m+1} & -J_{m+1, m+2} & -J_{m+1, n} \\
-J_{m+2,1} & -J_{m+2, m} & -J_{m+2, m+1} & \lambda+k^{2} D_{m+2}-J_{m+2, m+2} & -J_{m+2, n} \\
\vdots & \vdots & \vdots & \vdots & \vdots
\end{array}\right) .
$$

Case 1 For solutions of the dispersion relation satisfying $\lambda_{*}\left(k^{2}\right) \sim O(1)$ as $k^{2} \rightarrow \infty$, (15) reduces to

$$
0=k^{2(n-m)}\left(\prod_{q=m+1}^{q=n} D_{q}\right) \operatorname{det}(\lambda \mathbf{I}-\hat{\mathbf{J}})\left(1+O\left(\frac{1}{k^{2}}\right)\right),
$$


where

$$
\hat{\mathbf{J}}=\left(\begin{array}{ccc}
J_{11} & \cdots & J_{1 m} \\
\vdots & & \vdots \\
J_{m 1} & \cdots & J_{m m}
\end{array}\right)
$$

is the Jacobian associated with the receptor kinetics. Hence, we have $m$ roots of the dispersion relation for $k$ sufficiently large, given by the eigenvalues of the Jacobian associated with the receptor dynamics.

Case 2 We consider potential roots of the dispersion relation of the form $\lambda_{*}\left(k^{2}\right)=$ $\mu k^{2}$ with $\mu \sim O(1)$ as $k^{2} \rightarrow \infty$. The dispersion relation becomes

$$
0=k^{2 n} \mu^{m}\left\{\prod_{q=m+1}^{n}\left(\mu+D_{q}\right)\right\}\left(1+O\left(\frac{1}{k^{2}}\right)\right),
$$

which yields $n-m$ approximate roots associated with $\mu \approx-D_{q}, q \in\{m+1, \ldots, n\}$, and thus

$$
\lambda_{*}\left(k^{2}\right) \approx-k^{2} D_{m+1},-k^{2} D_{m+2}, \ldots,-k^{2} D_{n} .
$$

The combination of Case 1 and Case 2 reveals $n$ solutions for the dispersion relation, which has no further roots as it is a polynomial of degree $n$ in $\lambda$. Therefore, in the presence of receptors, the constraint that the system is stable to sufficiently high wavenumbers in order to prevent a breakdown of the continuum approximation requires that the Jacobian of the receptor kinetics has negative eigenvalues. In other words, the spatially homogeneous receptor dynamics must be linearly stable. In the special case of one receptor, we therefore require that it is self-inhibitory. Such constraints are not apparent when all species are freely diffusing and thus are a distinct feature of incorporating receptor dynamics. Finally, note that analogous comments immediately apply for intracellular protein dynamics.

\section{Exploration of a Proposed Patterning Network}

A detailed analysis of the biological interactions that lead to the emergence of hair follicle patterns in mouse is given by Mou et al. (2006). Moreover, the authors propose a model for the generation of the primary hair follicle pattern based on the interactions of three species: a non-diffusing, generic receptor Edar $(E)$, connective tissue growth factor CTGF $(C)$, and bone morphogenetic protein BMP $(B)$. The model is based on the following observations: (1) Edar shows rapid positive feedback (which is thought to be crucial for the patterning mechanism) and induces activation of both BMP and CTGF; (2) production of Edar is regulated by BMP through inhibition; (3) BMP is allowed to act only at a distance from its site of synthesis. This corresponds to long-range diffusion of BMP and short-range diffusion of CTGF. The proposed model can be described as in Fig. 1(a). 


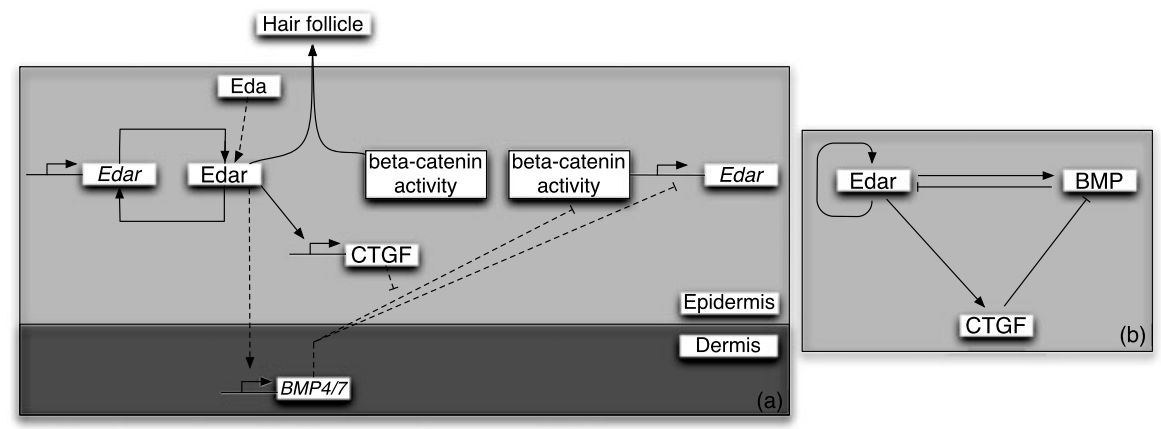

Fig. 1 (a) The model proposed by Mou et al. (2006). (b) The corresponding diagram of molecular interactions proposed to give rise to patterning

\subsection{Model for Hair Follicle Patterning}

The interactions of these three species are summarised in Fig. 1(b) and can be described by the following system of partial differential equations (PDEs):

$$
\begin{aligned}
& \frac{\partial E}{\partial t}=F(E, B), \\
& \frac{\partial C}{\partial t}=G(E, C)+D_{C} \nabla^{2} C, \\
& \frac{\partial B}{\partial t}=H(E, C, B)+D_{B} \nabla^{2} B,
\end{aligned}
$$

where the functions $F, G$ and $H$ describe the kinetics of the molecular interactions, and $D_{C}$ and $D_{B}$ are the diffusion coefficients of CTGF and BMP, respectively.

From Sect. 2, we know that in a system where there is one non-diffusible component, this component needs to down-regulate itself in the vicinity of the stationary state to prevent instabilities on arbitrarily small scales and a breakdown of the continuum approximation. Thus, the model proposed by Mou et al. (2006) for primary hair follicle patterning based on the presence of a Turing instability cannot lead to patterning. This is because Edar, the supposed key driver in the generation of patterning is fixed in cells and cannot diffuse, and Edar undergoes local auto-regulation and signal amplification.

Even when the kinetic terms of the model are modified to meet the required condition for large wavenumber behaviour, i.e. $\partial F / \partial E<0$ at the homogeneous steady state, a stable pattern still cannot be formed, as we shall now show.

The observed interactions among Edar, CTGF and BMP hypothesised by Mou et al. (2006), with the required modification that Edar is self-inhibitory, can be characterised using the following conditions on the kinetic terms evaluated at the stationary state; see Fig. 1(b):

$$
\begin{aligned}
& D_{C}>0, \quad D_{B}>D_{C}, \quad F_{E}<0, \quad F_{B}<0, \\
& H_{E}>0, \quad H_{C}<0, \quad G_{E}>0 .
\end{aligned}
$$


Here, the subscripts of kinetic functions $F, G, H$ represent partial derivatives evaluated at the steady state, for instance, $F_{E}:=\partial F / \partial E, D_{C}$ and $D_{B}$ denote diffusion coefficients of CTGF and BMP, respectively.

Given the reaction-diffusion system described by (20), the dispersion relation (8) takes the following form:

$$
\begin{aligned}
p\left(\lambda, k^{2}\right)= & \operatorname{det}\left(\lambda \mathbf{I}+k^{2} \mathbf{D}-\mathbf{J}\right) \\
= & -F_{E} G_{C} H_{B}-F_{B} G_{E} H_{C}+F_{B} G_{C} H_{E} \\
& +\left[\left(D_{B} F_{E} G_{C}+D_{C} F_{E} H_{B}-D_{C} F_{B} H_{E}\right) k^{2}-D_{B} D_{C} F_{E} k^{4}\right] \\
& +\lambda\left[F_{E} G_{C}+F_{E} H_{B}+G_{C} H_{B}-F_{B} H_{E}\right. \\
& \left.+\left(-D_{B} F_{E}-D_{C} F_{E}-D_{B} G_{C}-D_{C} H_{B}\right) k^{2}+D_{B} D_{C} k^{4}\right] \\
& +\lambda^{2}\left[-F_{E}-G_{C}-H_{B}+\left(D_{B}+D_{C}\right) k^{2}\right]+\lambda^{3} .
\end{aligned}
$$

Noting we have $F_{E}<0$ and thus stability at large wavenumbers, the Turing instability can occur once two conditions are met: the homogeneous steady state is (1) stable in the absence of diffusion and (2) unstable for finite wavenumbers $k^{2}>0$ associated with eigenvalues of the Laplacian and its boundary conditions.

The first condition, stability of the spatially uniform steady state, can be equivalently stated using the Routh-Hurwitz conditions which in our case (20) read:

$$
\begin{aligned}
-\left(F_{E}+G_{C}+H_{B}\right) & >0, \\
-F_{E} G_{C} H_{B}-F_{B} G_{E} H_{C}+F_{B} G_{C} H_{E} & >0, \\
F_{B} G_{E} H_{C}-\left(F_{E}+H_{B}\right)\left(\left(F_{E}+G_{C}\right)\left(G_{C}+H_{B}\right)-F_{B} H_{E}\right) & >0,
\end{aligned}
$$

where all terms are evaluated at the spatially uniform steady state, $\mathbf{u}^{*}=\left(E^{*}, C^{*}, B^{*}\right)$. After some algebraic manipulation, it can be shown that these constraints can be satisfied only if $G_{C}<0$, a necessary condition. Thus, we see that $H_{B}$ has an upper limit. Notice that the terms $G_{C}$ and $H_{B}$ represent self-regulation of CTGF and BMP, respectively, via mechanisms such as degradation or auto-catalysis. Thus, CTGF is required to be down-regulated by itself near the stationary state.

To determine whether the spatially uniform stationary state becomes unstable for non-zero wave-numbers, $k^{2}>0$, one finds $\lambda_{*}\left(k^{2}\right)$, the roots of the dispersion relation, $p\left(\lambda, k^{2}\right)=0$, for any given $k^{2}>0$. If $\operatorname{Re}\left(\lambda_{*}\left(k^{2}\right)\right)>0$ for any $k^{2}>0$ that is also minus an eigenvalue of the Laplacian, the system is linearly unstable. However, finding $\lambda_{*}\left(k^{2}\right)$ via the dispersion relation is difficult, even in the case when there are three interacting species and $p\left(\lambda, k^{2}\right)$ is a cubic polynomial.

To proceed, note that

$$
0=\frac{\mathrm{d} p}{\mathrm{~d} k^{2}}\left(\lambda_{*}\left(k^{2}\right), k^{2}\right)
$$

as $p\left(\lambda_{*}\left(k^{2}\right), k^{2}\right)=0$ for all $k^{2}$. Given $\operatorname{Re}\left(\lambda_{*}\left(k^{2}\right)\right)<0$ for $k^{2}=0$, by stability of the homogeneous steady state and for $k^{2}$ sufficiently large, an instability requires that 
$\operatorname{Re}\left(\lambda_{*}\left(k^{2}\right)\right)>0$ at a turning point in $\operatorname{Re}\left(\lambda_{*}\left(k^{2}\right)\right)$ as $k^{2}$ is varied. This is analytically more tractable as

$$
\left.\frac{\mathrm{d} p}{\mathrm{~d} k^{2}}\left(\lambda_{*}\left(k^{2}\right), k\right)\right|_{\frac{\mathrm{dRe}\left(\lambda_{*}\left(k^{2}\right)\right)}{\mathrm{d} k^{2}}=0}=0,
$$

yields quadratic polynomials for $\lambda_{*}\left(k^{2}\right)$ in our case.

\subsubsection{Case 1: $\operatorname{Im}\left(\lambda_{*}\left(k^{2}\right)\right)=0$}

Let $\lambda_{* *}$ denote the value of $\lambda_{*}\left(k^{2}\right)$ at the turning point. We have

$$
\begin{aligned}
0= & \left.\frac{\mathrm{d} p}{\mathrm{~d} k^{2}}\left(\lambda_{*}\left(k^{2}\right), k\right)\right|_{\frac{\mathrm{dRe}\left(\lambda_{*}\left(k^{2}\right)\right)}{\mathrm{d} k^{2}}=0} \\
= & \underbrace{D_{B} F_{E} G_{C}}_{>0}+D_{C} F_{E} H_{B} \underbrace{-D_{C} F_{B} H_{E}}_{>0} \underbrace{-2 D_{B} D_{C} F_{E} k^{2}}_{>0} \\
& +\underbrace{(\underbrace{\left(D_{B}+D_{C}\right)}_{>0}\left(-F_{E}\right)}_{>0} \underbrace{-D_{B} G_{C}}_{>0}-D_{C} H_{B}+\underbrace{2 D_{B} D_{C} k^{2}}_{>0}) \lambda_{* *} \\
& +\underbrace{\left(D_{B}+D_{C}\right)}_{>0} \lambda_{* *}^{2},
\end{aligned}
$$

where the inequalities follow from (21) and the Routh-Hurwitz conditions (23). Using Descartes' rule of signs, one can easily show that $H_{B}>0$ since otherwise $\lambda_{* *}<0$. One can further show that, even when $H_{B}>0$, all the coefficients in (26) are positive via further use of Descartes' rule of signs, once all the Routh-Hurwitz conditions are met together with (21) and (23).

Thus, in summary, when considering real solutions of the dispersion relation, which correspond to non-oscillatory self-organisation, the Turing instability cannot occur.

\subsubsection{Case $2: \operatorname{Im}\left(\lambda_{*}\left(k^{2}\right)\right) \neq 0$}

The more general case, when $\lambda_{*}\left(k^{2}\right)$ has non-zero imaginary part, needs more careful attention. However, it is still possible to show that there is no Turing instability for $H_{B}<0$ using an analogous procedure to the preceding one. Again, a Turing instability requires $\operatorname{Re}\left(\lambda_{*}\left(k^{2}\right)\right)>0$ at its turning point.

Since we have already considered real roots, we focus on complex roots of the dispersion relation and, in particular, note that complex roots occur in conjugate pairs. For $\lambda_{* *}$, a complex solution of the dispersion relation at a turning point of $\operatorname{Re}\left(\lambda_{*}\left(k^{2}\right)\right)$, let the two conjugate roots be denoted by

$$
\lambda_{* *, 1}=\mu_{* *}+i \nu_{* *}, \quad \lambda_{* *, 2}=\mu_{* *}-i \nu_{* *} .
$$

If these expressions are substituted back into the derivative of the dispersion relation, (25), at a turning point of $\operatorname{Re}\left(\lambda_{*}\left(k^{2}\right)\right)$ the resulting implicit relation for the 
unknown real part of $\lambda, \operatorname{Re}\left(\lambda_{* *}\right)=\mu_{* *}$, contains not only the imaginary part $v_{* *}$ but also

$$
v_{* *}^{\prime}:=\left.\frac{\mathrm{d} \nu}{\mathrm{d} k^{2}}\right|_{\nu=v_{* *}} .
$$

Instead of substituting these conjugate roots into the derivative of the dispersion relation (25), where we would have to deal with the term $v_{* *}^{\prime}$, we consider the difference of the dispersion relation (22) for the conjugate pair of roots, and Vieta's relations

$$
\begin{aligned}
0= & \frac{1}{2 i v_{* *}}\left(\left.p\left(\lambda_{* *}, k^{2}\right)\right|_{\lambda_{* *}=\mu_{* *}+i v_{* *}}-\left.p\left(\lambda_{* *}, k^{2}\right)\right|_{\lambda_{* *}=\mu_{* *}-i v_{* *}}\right) \\
= & \underbrace{F_{E} G_{C}+F_{E} H_{B}+G_{C} H_{B}-F_{B} H_{E}}_{>0} \\
& +\underbrace{\left(-D_{B} F_{E}-D_{C} F_{E}-D_{B} G_{C}-D_{C} H_{B}\right) k^{2}}_{>0}+\underbrace{D_{B} D_{C} k^{4}}_{>0} \\
& +\underbrace{\left(-2 F_{E}-2 G_{C}-2 H_{B}+\left(2 D_{B}+2 D_{C}\right) k^{2}\right)}_{>0} \mu_{* *}+3 \mu_{* *}^{2}-v_{* *}^{2},
\end{aligned}
$$

where the inequalities hold from the Routh-Hurwitz conditions (23).

Notice the only negative term that may lead to the existence of positive $\mu_{* *}$ is the negative square of the imaginary part, $v_{* *}$. To get the final conclusion as to the existence of $\mu>0$ for $H_{B}<0$ at a turning point, we need to find a relation between the real and imaginary parts of $\lambda, \mu$ and $\nu$. This can be easily deduced from Vieta's relations and, if substituted back into the equation for $\mu$ (29), it can be shown that all the coefficients in this relation are positive again once we require $H_{B}<0$, and the Routh-Hurwitz conditions together with the required and necessary conditions. Thus, for $H_{B}<0$ there can be no Turing pattern formation since all the possible values of $\operatorname{Re}(\lambda)=\mu$ are negative for any wave-number, $k^{2}$.

However, when $H_{B}>0$ a Turing instability can occur. Classifying the unstable region of parameter space is not insightful given the analytical complexity. Instead, we simply seek parameter values which exhibit a Turing instability, or rather a TuringHopf instability, as here only temporally oscillating instabilities are possible.

If Vieta's relations are substituted into (29), we obtain a cubic polynomial in $\mu$ where the only negative term is $2 H_{B}\left[D_{B}\left(F_{E}+G_{C}\right)+D_{C}\left(F_{E}+G_{C}\right)\right]$ in the coefficient of $\mu^{1}$. From Descarte's rule of signs, this term needs to dominate to induce a root with positive real part. Motivated by this observation, we assumed further constraints on the kinetic terms and diffusion coefficients to simplify the dispersion relation. In this way, it is possible to find a set of parameter values that lead to a Turing-Hopf instability. If we assume $D_{C} \ll 1, H_{B} \ll 1$, many terms can be neglected and the following example reveals oscillatory self-organisation:

$$
\begin{aligned}
& D_{C}=10^{-8}, \quad D_{B}=10^{-3}, \quad F_{E}=-4, \quad F_{B}=-19, \quad G_{E}=\frac{21}{512}, \\
& G_{C}=-1, \quad H_{E}=1, \quad H_{C}=-1, \quad H_{B}=\frac{9}{2} .
\end{aligned}
$$




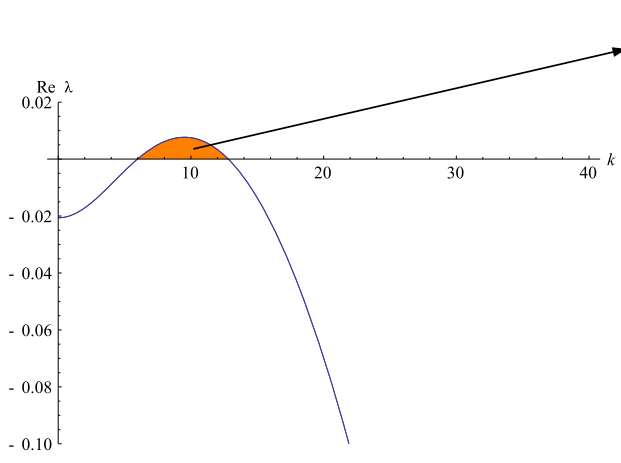

a)

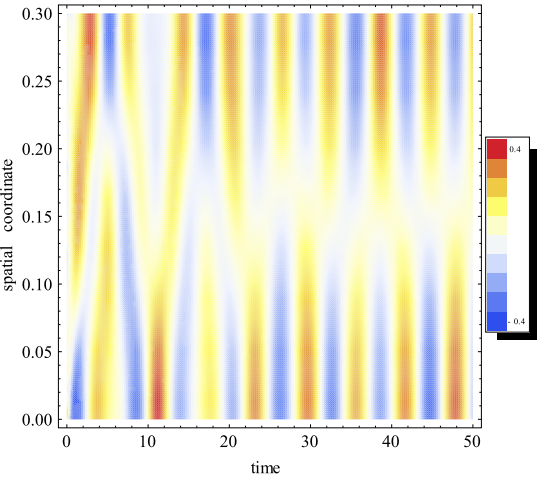

b)

Fig. 2 (Colour online) Even when the model with network structure predicted by Mou et al. (2006) is modified to fit the constraints found in this work $\left(F_{E}<0, G_{C}<0, H_{B}>0\right)$ emergent self-organisation is only possible via an oscillatory Turing-Hopf bifurcation. The example from (30) demonstrates theoretically found results. (a) The real part of the dispersion relation's roots. (b) Instability occurs once the critical region of wavenumbers is reached. Notice the temporal variation of the pattern $\operatorname{as} \operatorname{Im}(\lambda) \neq 0$

The dispersion relation behaves as expected and numerical solution of the PDE system shows the emergence of spatio-temporal pattern, as illustrated in Fig. 2.

To summarise, even when the model with experimentally predicted network structure $(F=F(E, B), G=G(E, C), H=H(E, C, B))$ is modified to fit the constraints found in this work $\left(F_{E}<0, G_{C}<0, H_{B}>0\right)$ emergent self-organisation is only possible via an oscillatory Turing-Hopf bifurcation. However, as mentioned previously, this type of patterning additionally requires the existence of a further mechanism to stop the temporal oscillations that are concomitant with the emergence of spatial structure. Moreover, if the desired interactions among Edar, CTGF, and BMP satisfy $D_{C}>0, D_{B}>D_{C}, F_{B}<0, H_{E}>0, H_{C}<0, G_{E}>0$, as suggested by Mou et al. (2006), then one necessarily requires $F_{E}<0, G_{C}<0, H_{B}>0$ for even this possibility of temporally oscillating spatial heterogeneity. Finally, we remark that if CTGF is omitted from the model, one can show that considering BMP and Edar in isolation does not give rise to patterning in a reaction diffusion model regardless of whether Edar is self-inhibitory or otherwise.

\subsection{CTGF Inhibits the Effect of BMP, Not BMP Production}

One can also interpret the kinetics of CTGF from the study by Mou et al. (2006) in a different way: CTGF could be considered to down-regulate "the effect" of BMP inhibition. In this context, one could consider CTGF binding to BMP, preventing its action, at least temporarily. Hence, in the presence of CTGF the gross BMP level does not drop, only its activity. In this scenario, the action of CTGF would be represented by CTGF down-regulation of the inhibition of Edar by BMP. This is because CTGF protein binds to and, at least temporarily, inactivates BMP protein in the extracellular space. Moreover, biochemical evidence suggests that CTGF should inhibit BMP's effects, not BMP production (Abreu et al. 2002).

The kinetic terms considered in the previous analysis did not include this interaction among Edar, CTGF and BMP. However, the action of CTGF down-regulating 
the inhibition of Edar by BMP can be represented by a term of the form

$$
F(E, C, B)=\cdots-k_{2} \frac{E B}{k_{3}+C}+\cdots,
$$

which entails the function $F$ should be monotonic increasing with levels of CTGF.

\subsubsection{One Example of a Turing Instability}

A stability analysis can be carried out using the same techniques though with the difficulty of increased generality, as $F=F(E, C, B), G=G(E, C), H=H(E, C, B)$. Nonetheless, the requirement remains that the non-diffusing species, Edar, must be self-inhibitory to avoid a breakdown of the continuum approximation. Further, it can be shown that if CTGF down-regulates the inhibition of Edar by BMP, a nonoscillatory Turing instability, whereby the roots of the dispersion relation are real, remains possible. To illustrate this, two simplifications are made: (1) BMP is not influenced directly by CTGF, $H_{C}=0$; and (2) BMP does not regulate itself, $H_{B}=0$. With these simplifications, the constraints imposed by the empirical observations of Mou et al. (2006), together with those imposed by a Turing stability with no temporal oscillation and stability at large wavenumbers, gives rises to the following:

1. $D_{C}>0, D_{B} \geq D_{C}, F_{B}<0, H_{E}>0, G_{E}>0, F_{C}>0$, imposed by empirical observation from Mou et al.'s (2006) study;

2. $H_{C}=H_{B}=0$, additionally imposed simplifications to facilitate the search for a Turing instability;

3. $F_{E}<0$, required from a consideration of the large wavenumber behaviour;

4. $G_{C}<0, F_{E} G_{C}-F_{C} G_{E}<0$ and $-F_{B} G_{C} H_{E}-\left(F_{E}+G_{C}\right)\left(F_{E} G_{C}-F_{C} G_{E}-\right.$ $\left.F_{B} H_{E}\right)>0$, a consequence of the Routh-Hurwitz conditions, for stability in the absence of diffusion;

5. $0<H_{E}<\frac{D_{B}}{D_{C}\left(-F_{B}\right)}\left(\sqrt{F_{C} G_{E}}-\sqrt{F_{E} G_{C}}\right)^{2}$, from the need for instability at a nonzero wavenumber.

Note that the final condition can always be satisfied for sufficiently large values of $D_{B} / D_{C}$ once the first four conditions are fulfilled.

To demonstrate the validity of our conclusions, and the existence of a Turing instability note firstly that the above constraints are satisfied by the following parameter choices:

$$
\begin{aligned}
& D_{C}=1, \quad D_{B}=10, \quad F_{E}=-0.5, \quad F_{C}=2.25, \quad F_{B}=-6, \\
& G_{E}=1, \quad G_{C}=-1, \quad H_{E}=1, \quad H_{C}=0, \quad H_{B}=0 .
\end{aligned}
$$

Furthermore, a simulation of the linearised PDEs corresponding to this parameter regime exhibits the Turing instability; see Fig 3.

\subsubsection{A Second Example of a Turing Instability Without the Need for Short-Range Activation and Long-Range Inhibition}

The previous example was obtained in the simplified setting $H_{B}=H_{C}=0$, where the analysis led to the conclusion that a large enough ratio $D_{B} / D_{C}$ is needed in order to obtain stable pattern through Turing instability; see Sect. 3.2.1. 


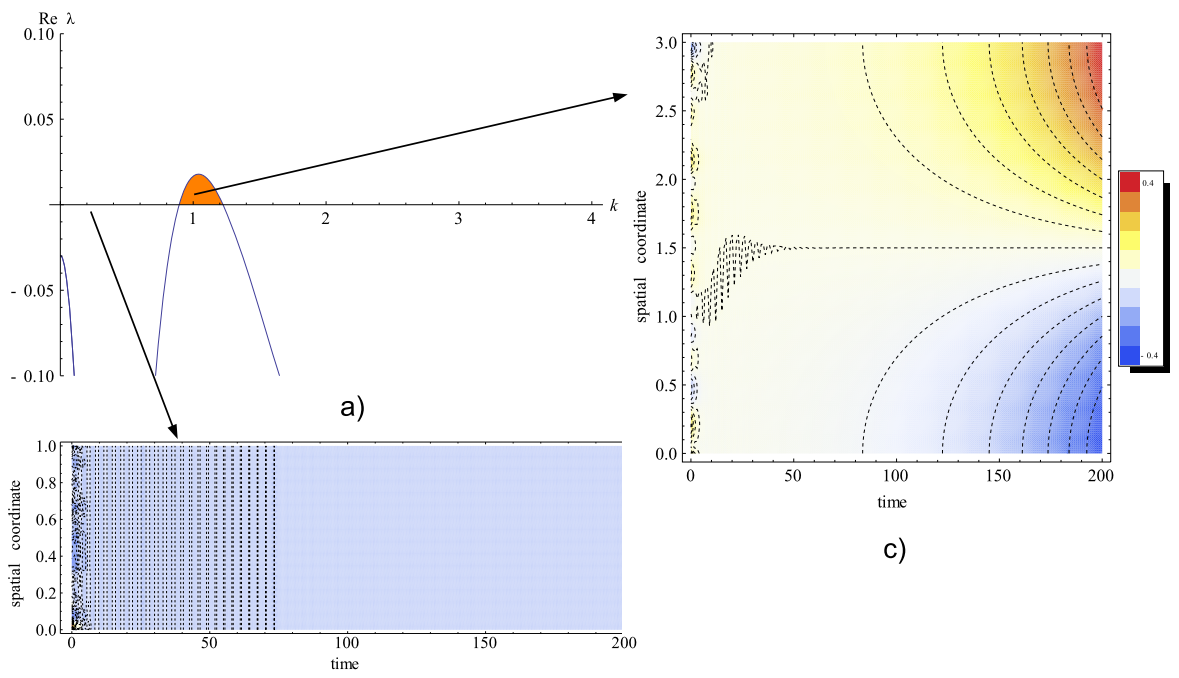

b)

Fig. 3 (Colour online) If the interactions between CTGF and BMP from Mou et al.'s empirical model are interpreted differently, diffusion-driven pattern formation can take place. (a) The real part of the dispersion relation's roots for the case in which $H_{B}=0$. (b), (c) Example simulations for parameter values: (b) a stable case with low wavenumbers, (c) a diffusion-driven instability occurs. Notice that the size of the domain restricts pattern formation. Parameter values are as given in (32)

However, a Turing instability can also arise even without the standard demand for short-range activation and long-range inhibition which stems from analysis of the two-species model. In the context of the constraints from Mou et al. (2006), though without the simplifying imposition of $H_{C}=H_{B}=0$, consider the following parameter set:

$$
\begin{aligned}
& D_{C}=1, \quad D_{B}=1, \quad F_{E}=-6, \quad F_{C}=5, \quad F_{B}=-\frac{7}{2}, \\
& G_{E}=\frac{479}{64}, \quad G_{C}=-4, \quad H_{E}=1, \quad H_{C}=0, \quad H_{B}=-1 .
\end{aligned}
$$

In particular, note the diffusion coefficients are equal so that there is no differential range of the diffusible signals. The corresponding dispersion relation highlighting the existence of the Turing instability can be seen in Fig. 4.

\subsection{The Dangers of Model Reductionism}

These studies of the interactions within hair follicle patterning (Mou et al. 2006) also highlight more general difficulties in the use of reductionism in modelling morphogen networks. We now outline our claim in more detail.

\subsubsection{The Three-Species Model Does Not Pattern but the Two-Species, Reduced Model Does}

There are three possible reasons why patterning due to a Turing instability does not occur: (1) instability of the spatially uniform stationary state; (2) a structural insta- 


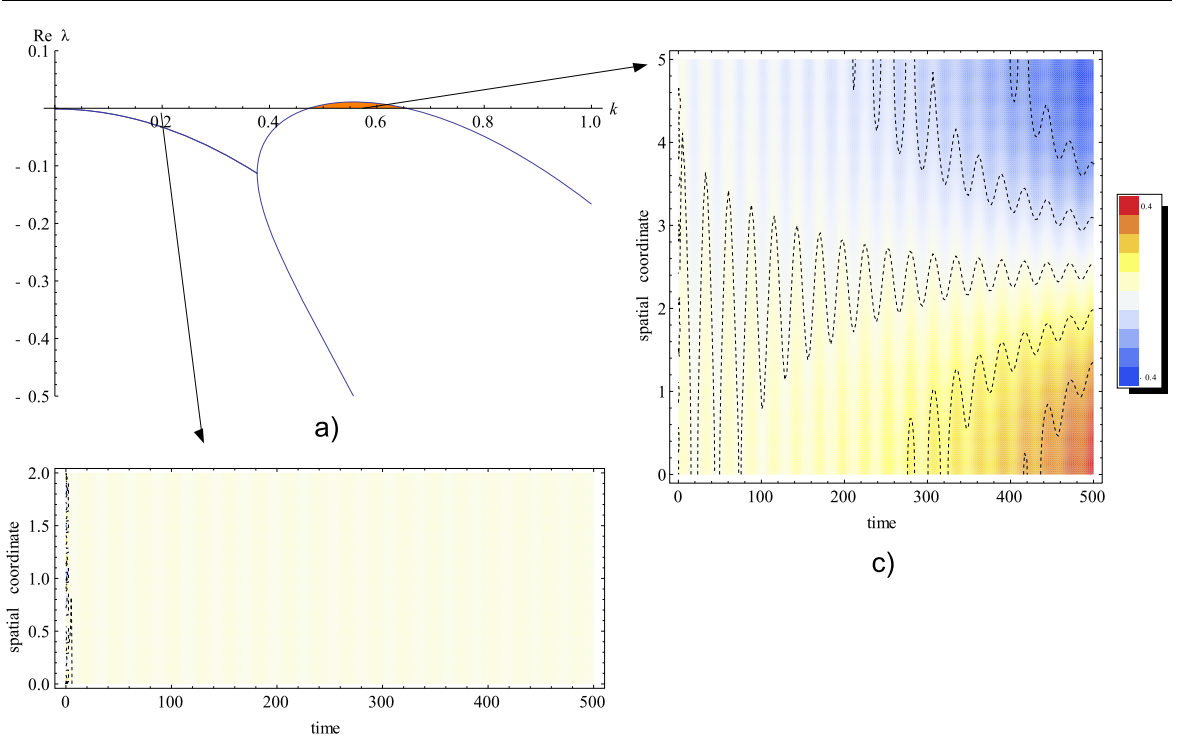

b)

Fig. 4 (Colour online) In the three-species model shown in Fig. 3, a Turing instability may occur even without short-range activation and long-range inhibition. In this figure, an example resulting in pattern formation even when diffusion coefficients are equal is presented. Parameters are given in (33). (a) The real parts of the dispersion relation roots for the case in which $H_{B}=0$. (b), (c) Numerical solutions of the system. Notice that the size of the domain restricts pattern formation

bility or continuum approximation breakdown (as discussed in Sect. 2.1.1); and (3) no instability occurs for non-zero wavenumbers, $k^{2}$. We will show that for all these cases one can find many examples when three three-species model does not pattern whereas the two-species, reduced model does.

Instability of the Spatially Uniform Stationary State The stationary state in a twospecies representation of the model is stable once it fulfils the Routh-Hurwitz conditions, namely $-\left(G_{C}+H_{B}\right)>0, G_{C} H_{B}-G_{B} H_{C}>0$, where we use the notation from the three-species model discussed in this work (considering only the two non-diffusing species). This stable stationary state can very easily become unstable in the corresponding three-species model. In particular, one necessary condition reads $-\left(F_{E}+G_{C}+H_{B}\right)>0$, and this can be violated simply by large enough $F_{E}>-\left(G_{C}+H_{B}\right)>0$.

A Structural Instability Consider a system of diffusible morphogens undergoing a Turing instability via the vanilla mechanism of short-range activation and long-range inhibition, and introduce a non-diffusing receptor that interacts with this system and possesses auto-catalytic dynamics. The three component system will destabilise on arbitrarily small lengthscales (this holds for any system since the necessary condition for the additional non-diffusing species is independent of the interactions between other species, as shown in Sect. 2.1.1). The result is a breakdown of the continuum approximation, whereas a two component approximation of the system, neglecting 
receptor dynamics, will yield a system with large scale patterning. In other words, whatever the interactions between the two diffusing species, there can be no stable pattern formation once a non-diffusive morphogen with auto-catalytic regulation is added to the system (due to structural instability). Thus, if the kinetics between the two diffusing species are such that they meet the classical two-species Turing instability conditions, we see that the three-species system, which does not pattern, is reduced by omitting the non-diffusive species (and the accompanying interactions) into a two-species model that does pattern.

No Instability for Non-zero Wavenumbers The network structure of the system itself can be such that there is no Turing driven instability in the three-species system (and not due to a breakdown of the continuum approximation) and still the two-species reduced model does pattern; see Fig. 6 and the parameter values are listed below:

$$
\begin{aligned}
& D_{E}=0, \quad \underline{D_{C}=\frac{1}{4}}, \quad \underline{D_{B}=1}, \\
& F_{E}=-3, \quad F_{C}=24, \quad F_{B}=-45, \\
& G_{E}=-4, \quad \underline{G_{C}=1}, \quad \underline{G_{B}=-\frac{17}{8}}, \\
& H_{E}=-1, \quad \underline{H_{C}=1}, \quad \underline{H_{B}=-2} .
\end{aligned}
$$

One can easily check that the two-species system $G, B$ with the parameters underlined in (34) has a stationary steady state, and for

$$
k^{2} \in\left(1-\frac{1}{\sqrt{2}}, 1+\frac{1}{\sqrt{2}}\right)
$$

instability occurs as $\lambda>0$. Meanwhile, the dispersion relation for the three-species model with parameter values from (34) is

$$
p\left(\lambda, k^{2}\right)=\frac{51}{8}+\frac{333 k^{2}}{4}+\frac{3 k^{4}}{4}+\left(\frac{433}{8}+\frac{13 k^{2}}{4}+\frac{k^{4}}{4}\right) \lambda+\left(4+\frac{5 k^{2}}{4}\right) \lambda^{2}+\lambda^{3},
$$

which has all coefficients positive and hence no real positive root. Moreover, it can be shown that even the real part of the conjugate roots remain negative for all $k^{2}>0$. Notice that $F_{E}<0$, thereby guaranteeing stability for large wavenumbers.

\subsubsection{The Three-Species Model Does Pattern and Two-Species Reduced Model Does Not}

In contrast, consider the Edar, CTGF, and BMP system where self-organisation is observed, as in example (33) of Sect. 3.2. If a modelling simplification is considered with a neglect of the non-diffusing receptor Edar, leaving only the interactions of CTGF and BMP, no spatial heterogeneity would emerge, as they have equal diffusion coefficients which prevents a Turing instability. Further, consider a three-species system, as discussed in Sect. 3.2.1, that leads to Turing instability. A typical two-species 
Fig. 5 (Colour online) (b) A typical simplification of the interactions among three species represented in (a) on neglecting the non-diffusing receptor. However, these two models possess a very different patterning behaviour
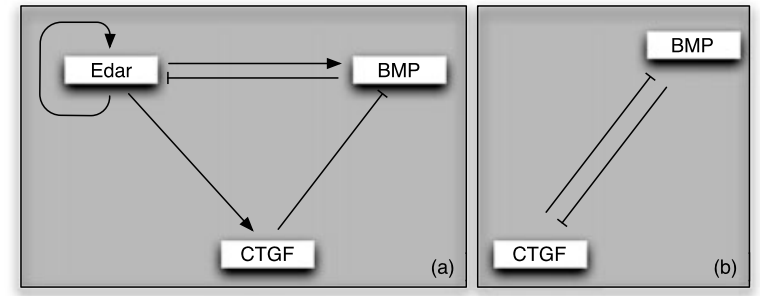

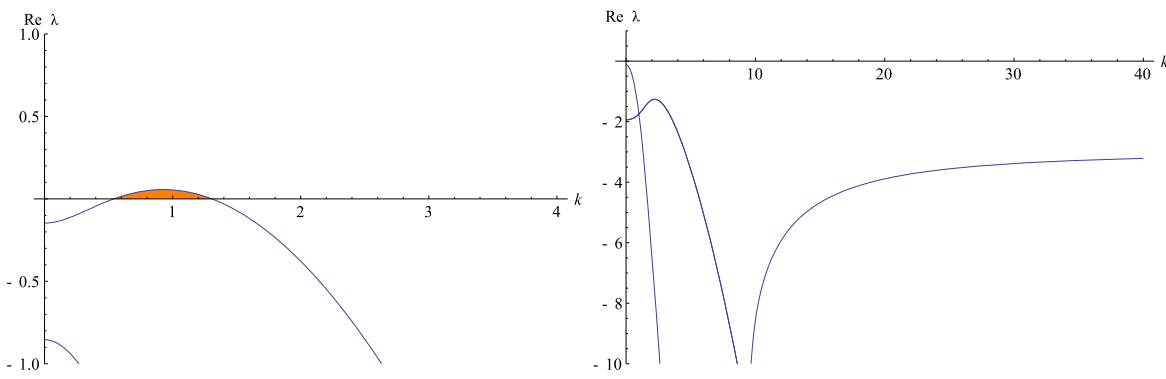

a)

b)

Fig. 6 (Colour online) The real parts of the dispersion relation roots associated with the parameter values listed in (34). These document the disparity of (a) the reduced model leading to pattern formation with the underlined parameter values listed in (34) and (b) the converse prediction from a model with an extra species that is stable for all wave-numbers

reduction of such a system (as might be the case when unaware of the presence of a receptor mediator) would be carried out as described in Fig. 5. However, one can check that this simplification does not lead to pattern formation via a diffusion-driven instability since $G_{B}<0$ and $H_{C}<0$ which contradicts the necessary conditions for a Turing instability in two-species model as the latter context requires an opposite sign for these two terms.

Thus, we have two examples when a two-species Turing model approximation gives opposing predictions to those emerging from networks with more species, further highlighting that reducing the interacting dynamics of diffusible morphogens and their receptors is potentially fraught with difficulty.

\section{Discussion and Conclusions}

We have explored the influence of non-diffusible receptor dynamics on the prospect of spontaneous self-organisation within networks of interacting morphogens, firstly in a general context and then specifically within the framework for hair follicle prepatterns suggested by Mou et al.'s (2006) experiments. In this framework, a key regulatory step is the suppression of production of a receptor, Edar, by diffusible BMP. In addition to the skin culture experiments described by Mou et al. (2006), similar phenotypes of mice with increased Edar production (Mou et al. 2008) and those with 
reduced BMP activity (Sharov et al. 2006; Plikus et al. 2008) highlight the importance of the BMP-Edar regulatory link. However, it is likely that other molecular systems also operate to pattern hair follicles, particularly given the diversity of hair follicle types which form at different times and at distinct locations during mammalian embryonic development. For example, Sick et al. (2006) suggested that secreted ligands of the WNT family act as activators during hair follicle pre-patterning, while secreted DKK proteins, which compete with WNT for binding to the same receptor, act as inhibitors. It is likely that the Edar-BMP and WNT-DKK systems are interlinked based on emerging cross-regulation observed between these pathways, with Edar signalling promoting WNT production and vice versa (Zhang et al. 2009). These findings suggest the presence of multiple interlocked regulatory loops that may be important for patterning at least certain types of hair follicle.

The requirement of stability at arbitrarily large wavenumbers to ensure that there is no prospective breakdown of the continuum approximation due to instabilities at ever decreasing lengthscales is redundant for systems of interacting and diffusing morphogens. However, in the presence of non-diffusing species, for instance receptors which are not subject to transport over the lengthscales of long wavelength patterning and act in a cell autonomous manner, one finds that the spatially homogeneous interactions of the non-diffusing species must be stable. This is a non-trivial constraint, for instance highlighting that a single receptor in a morphogen network needs to be self-inhibitory. Furthermore, this emphasises that interactions can be non-trivially constrained without the need for detailed parameter estimation or experimentation by considering the dynamics of the bifurcation to spatial heterogeneity within general systems. In particular, once receptors or other non-diffusing species are considered, the constraints are even more stringent. While the main focus of the paper has been receptor dynamics, it is clear that the analyses and conclusions are also applicable for considering the coupling between intra-cellular protein dynamics and a network of interacting and diffusing morphogens.

We additionally considered a specific and empirically led example of hair follicle pre-patterning based on genetic and embryological studies. Here, we have further demonstrated that network structure alone, without the need for detailed specification of the kinetic interactions or parameter sweeps, can be used to determine the feasibility of a structural model, as well as providing constraints on possible interactions. Further demanding constraints emerge on requiring that spatial heterogeneity is not driven by temporally oscillating morphogen profiles as the system evolves away from the homogeneous steady state. Significant temporal oscillations of gene activity and expression are unlikely to occur during hair follicle pre-patterning due to the acquisition of definitive hair follicle cell fate during this process. Cell lineage tracing studies show that upon activation of the gene $S h h$, which appears to be controlled by a threshold of Edar activity (Pummila et al. 2007), cells irreversibly commit to a hair follicle fate and do not revert to non-follicle (i.e. epidermal) fate (Levy et al. 2005). This "locking in" of activated regions in the pattern upon local attainment of a threshold level of activator would avoid oscillations across the entire field. The restrictions we describe enable a focussing of resources, both modelling and experimental, by reducing the morphogen and receptor interactions that need be considered for elucidating how self-organisation emerges, as well as providing testable hypotheses that are not contingent on the detailed interactions. 
We have also highlighted that, within morphogen networks with receptor dynamics, one does not require the classical picture of short-range activation and longrange inhibition for the induction of self-organisation, and that spatial heterogeneity can emerge with equal diffusion coefficients even within a model with only two diffusible components. This offers a potential resolution of a further difficulty with Turing's picture, namely the reconciliation of the need for differing diffusion rates with biomolecular diffusion given that, in practice the mechanism is predicted to require radically different transport coefficients for self-organisation in the absence of parameter fine-tuning (Baker et al. 2008). Similar observations have also emerged from the limited studies coupling intra-cellular protein dynamics to interacting and diffusing morphogens (Rauch and Millonas 2004). Consequently, there is the possibility that a search for a Turing pair of morphogens may be misdirected, in that the characteristic features of patterning formation in two-species models does not reflect that of larger networks. Furthermore, we have also highlighted, that taking even a system of two interacting morphogens and a single receptor, a naive model reduction to a vanilla Turing model can yield radically different modelling predictions. This emphasises the fundamental difficulties with model reductionism within biological self-organisation, especially the concept that Turing's mechanism can simply be considered as a caricature of larger biological networks. Thus, the vanilla Turing model not only suffers from extreme sensitivity, albeit ameliorated in many cases, but not all, by domain growth; it also can be a very misleading surrogate for the complex dynamics of real biological systems.

Acknowledgements This publication was based on work supported in part by Award No KUK-C1-01304, made by King Abdullah University of Science and Technology (KAUST). One of the authors (V.K.) is grateful for the hospitality of the Oxford Centre for Collaborative Applied Mathematics and the Institute for Mathematics and Its Applications during work on this project, as well as for many helpful comments and collaborations from his colleagues.

\section{References}

Abreu, J. G., Ketpura, N. I., Reversade, B., \& De Robertis, E. M. (2002). Connective-tissue growth factor (CTGF) modulates cell signalling by BMP and TGF- $\beta$. Nat. Cell Biol., 4(8), 599-604.

Alber, M., Tilmann, T. G., Hentschel, H. G. E., Kazmierczak, B., Zhang, Y., Zhu, J., \& Newman, S. A. (2008). The morphostatic limit for a model of skeletal pattern formation in the vertebrate limb. Bull. Math. Biol., 70(2), 460-483.

Alberts, B., Johnson, A., Walter, P., Lewis, J., Raff, M., \& Roberts, K. (2002). Molecular biology of the cell (5th ed.). New York: Garland Science.

Baker, R. E., Gaffney, E. A., \& Maini, P. K. (2008). Partial differential equations for self-organization in cellular and developmental biology. Nonlinearity, 21, R251-R290.

Bard, J., \& Lauder, I. (1974). How well does Turing's theory of morphogenesis work? J. Theor. Biol., 45, 501-531.

Bunow, B., Kernevez, J. P., Joly, G., \& Thomas, D. (1980). Pattern formation by reaction-diffusion instabilities: applications to morphogenesis in Drosophila. J. Theor. Biol., 84, 629-649.

Carroll, S. B. (2005). Evolution at two levels: on genes and form. PLoS Biol., 3(7), e245.

Chen, Y., \& Schier, A. F. (2002). Lefty proteins are long-range inhibitors of squint-mediated nodal signaling. Curr. Biol., 12, 2124-2128.

Crampin, E. J., Gaffney, E. A., \& Maini, P. K. (1999). Reaction and diffusion on growing domains: scenarios for robust pattern formation. Bull. Math. Biol., 61, 1093-1120.

Davidson, D. (1983). The mechanism of feather pattern development in the chick.1. the time of determination of feather position. J. Embryol. Exp. Morphol., 74, 245-259. 
Ermentrout, B., \& Lewis, M. (1997). Pattern formation in systems with one spatially distributed species. Bull. Math. Biol., 59, 533-549.

Gaffney, E. A., \& Monk, N. A. M. (2006). Gene expression time delays and Turing pattern formation system. Bull. Math. Biol., 68, 99-130.

Gierer, A., \& Meinhardt, H. (1972). A theory of biological pattern formation. Kybernetik, 12, 30-39.

Gilbert, S. F. (2006). Developmental biology (8th ed.). Sunderland: Sinauer.

Gregor, T., Bialek, W., de Ruyter van Steveninck, R., Tank, D., \& Wieschaus, E. (2005). Kinetics of morphogen gradient formation. Proc. Natl. Acad. Sci. USA, 102, 18403-18407.

Harris, M. P., Williamson, S., Fallon, J. F., Meinhardt, H., \& Prum, R. O. (2005). Molecular evidence for an activator-inhibitor mechanism in development of embryonic feather branching. Proc. Natl. Acad. Sci. USA, 102(33), 11734-11739.

Hentschel, H., Glimm, T., Glazier, J., \& Newman, S. (2004). Dynamical mechanisms for skeletal pattern formation in the vertebrate limb. Proc. R. Soc. Lond. B, Biol. Sci., 271(1549), 1713-1722.

Jung, H.-S., Francis-West, P. H., Widelitz, R. B., Jiang, T.-X., Ting-Berreth, S., Tickle, C., Wolpert, L., \& Chuong, C.-M. (1998). Local inhibitory action of BMPs and their relationships with activators in feather formation: implications for periodic patterning. Dev. Biol., 196(1), 11-23.

Kicheva, A., Pantazis, P., Bollenbach, T., Kalaidzidis, Y., Bittig, T., Jülicher, F., \& González-Gaitán, M. (2007). Kinetics of morphogen gradient formation. Science, 315, 521-525.

Leuzinger, S., Hirth, F., Gerlich, D., Acampora, D., Simeone, A., Gehring, W., Finkelstein, R., FurukuboTokunaga, K., \& Reichert, H. (1998). Equivalence of the fly orthodenticle gene and the human OTX genes in embryonic brain development of Drosophila. Development, 125(9), 1703-1710.

Levy, V., Lindon, C., Harfe, B. D., \& Morgan, B. A. (2005). Distinct stem cell populations regenerate the follicle and interfollicular epidermis. Dev. Cell, 9(6), 855-861.

Lin, C.-M., Jiang, T. X., Baker, R. E., Maini, P. K., Widelitz, R. B., \& Chuong, C.-M. (2009). Spots and stripes: pleomorphic patterning of stem cells via p-ERK-dependent cell chemotaxis shown by feather morphogenesis and mathematical simulation. Dev. Biol., 334(2), 369-382.

Lodish, H., Berk, A., Kaiser, C. A., Krieger, M., Scott, M. P., Bretscher, A., Ploegh, H., \& Matsudaira, P. (2007). Molecular cell biology (6th ed.). New York: Freeman.

Miura, T., \& Shiota, K. (2000a). Extracellular matrix environment influences chondrogenic pattern formation in limb bud micromass culture: experimental verification of theoretical models. Anat. Rec., 258, $100-107$.

Miura, T., \& Shiota, K. (2000b). TGF $\beta 2$ acts as an activator molecule in reaction-diffusion model and is involved in cell sorting phenomenon in mouse limb micromass culture. Dev. Dyn., 217, 241-249.

Miura, T., Shiota, K., Morriss-Kay, G., \& Maini, P. K. (2006). Mixed-mode pattern in Doublefoot mutant mouse limb-Turing reaction-diffusion model on a growing domain during limb development. J. Theor. Biol., 240, 562-573.

Moreo, P., Gaffney, E. A., Garcia-Aznar, J. M., \& Doblare, M. (2010). On the modelling of biological patterns with mechanochemical models: insights from analysis and computation. Bull. Math. Biol., 72(2), 400-431.

Mou, C., Jackson, B., Schneider, P., Overbeek, P. A., \& Headon, D. J. (2006). Generation of the primary hair follicle pattern. Proc. Natl. Acad. Sci. USA, 103(24), 9075-9080.

Mou, C., Thomason, H. A., Willan, P. M., Clowes, C., Harris, W. E., Drew, C. F., Dixon, J., Dixon, M. J., \& Headon, D. J. (2008). Enhanced ectodysplasin-a receptor (EDAR) signaling alters multiple fiber characteristics to produce the east Asian hair form. Human Mutat., 29(12), 1405-1411.

Mou, C., Pitel, F., Gourichon, D., Vignoles, F., Tzika, A., Tato, P., Yu, L., Burt, D. W., Bed'hom, B., Tixier-Boichard, M., Painter, K. J., \& Headon, D. J. (2011). Cryptic patterning of avian skin confers a developmental facility for loss of neck feathering. PLoS Biol., 9(3), e1001028.

Murray, J. D. (2003). Mathematical biology II: spatial models and biochemical applications (Vol. II, 3rd ed.). Berlin: Springer.

Plikus, M., Wang, W. P., Liu, J., Wang, X., Jiang, T. X., \& Chuong, C. M. (2008). Morpho-regulation of ectodermal organs: integument pathology and phenotypic variations in K14-Noggin engineered mice through modulation of bone morphogenic protein pathway. Am. J. Pathol., 164(3), 1099-1114.

Pummila, M., Fliniaux, I., Jaatinen, R., James, M. J., Laurikkala, J., Schneider, P., Thesleff, I., \& Mikkola, M. L. (2007). Ectodysplasin has a dual role in ectodermal organogenesis: inhibition of BMP activity and induction of Shh expression. Development, 134(1), 117-125.

Qian, H., \& Murray, J. D. (2001). A simple method of parameter space determination for diffusion-driven instability with three species. Appl. Math. Lett., 14, 405-411.

Rauch, E. M., \& Millonas, M. M. (2004). The role of trans-membrane signal transduction in Turing-type cellular pattern formation. J. Theor. Biol., 226, 401-407. 
Sakuma, R., Ohnishi, Y., Meno, C., Fujii, H., Juan, H., Takeuchi, J., Ogura, T., Li, E., Miyazono, K., \& Hamada, H. (2002). Inhibition of nodal signalling by lefty mediated through interaction with common receptors and efficient diffusion. Genes Cells, 7, 401-412.

Satnoianu, R. A., Menzinger, M., \& Maini, P. K. (2000). Turing instabilities in general systems. J. Math. Biol., 41, 493-512.

Schmidt-Ullrich, R., \& Paus, R. (2005). Molecular principles of hair follicle induction and morphogenesis. BioEssays, 27(3), 247-261.

Segel, L. A., \& Jackson, J. L. (1972). Dissipative structure-explanation and an ecological example. J. Theor. Biol., 37, 545-559.

Seirin-Lee, S., \& Gaffney, E. A. (2010). Aberrant behaviours of reaction diffusion self-organisation models on growing domains in the presence of gene expression time delays. Bull. Math. Biol., 72, 21612179 .

Seirin-Lee, S., Gaffney, E. A., \& Monk, N. A. M. (2010). The influence of gene expression time delays on Gierer-Meinhardt pattern formation systems. Bull. Math. Biol., 72, 2319-2360.

Seirin-Lee, S., Gaffney, E. A., \& Baker, R. E. (2011). The dynamics of Turing patterns for morphogen-regulated growing domains with cellular response delays. Bull. Math. Biol. doi:10.1007/ s11538-011-9634-8.

Sengel, P. (1990). Pattern formation in skin development. Int. J. Dev. Biol., 34(1), 33-50.

Sharov, A. A., Sharova, T. Y., Mardaryev, A. N., Tommasi di Vignano, A., Atoyan, R., Weiner, L., Yang, S., Brissette, J. L., Dotto, G. P., \& Botchkarev, V. A. (2006). Bone morphogenetic protein signaling regulates the size of hair follicles and modulates the expression of cell cycle-associated genes. Proc. Natl. Acad. Sci. USA, 103(48), 18166-18171.

Sick, S., Reinker, S., Timmer, J., \& Schlake, T. (2006). WNT and DKK determine hair follicle spacing through a reaction-diffusion mechanism. Science, 314(5804), 1447-1450.

Solnica-Krezel, L. (2003). Vertebrate development: taming the nodal waves. Curr. Biol., 13, R7-R9.

Turing, A. (1952). The chemical basis of morphogenesis. Philos. Trans. R. Soc. Lond. B, Biol. Sci., 237, 37-72.

White, K. A. J., \& Gilligan, C. A. (1998). Spatial heterogeneity in three species, plant-parasitehyperparasite, systems. Philos. Trans. R. Soc. B, 353, 543-557.

Wolpert, L. (1994). Positional information and pattern formation in development. Dev. Genet., 15(6), 485490.

Wolpert, L. (2002). Principles of development (2nd ed.). London: Oxford University Press.

Zhang, Y., Tomann, P., Andl, T., Gallant, N. M., Huelsken, J., Jerchow, B., Birchmeier, W., Paus, R., Piccolo, S., Mikkola, M. L., Morrisey, E. E., Overbeek, P. A., Scheidereit, C., Millar, S. E., \& SchmidtUllrich, R. (2009). Reciprocal requirements for EDA/EDAR/NF-kappaB and Wnt/beta-catenin signaling pathways in hair follicle induction. Dev. Cell, 17(1), 49-61. 